\title{
Spaces having no large dyadic subspace
}

\section{Jason Gait}

Gillman-Henriksen have defined a class of spaces, containing the discrete spaces and their Stone-Čech compactifications, called $F^{\prime}$-spaces. The dyadic spaces are the continuous images of products of finite discrete spaces - a class which contains the compact metric spaces and all compact topological groups. In this paper it is shown that $F^{\prime}$-spaces have no infinite dyadic subspaces and, almost always, no dyadic compactifications. An interesting corollary is that if $\beta X \backslash X$ is dyadic, then $X$ is pseudocompact.

The purpose of this paper is to investigate the relationship between dyadic spaces and the $F^{\prime}$-spaces of Gillman-Henriksen. Nearly all $F^{\prime}$-spaces are shown to be without large dyadic subspaces, and without dyadic compactifications. This fact is exploited to see that the dyadicity of either $\beta X$ or $\beta X \backslash X$ determines that $X$ is pseudocompact, while the more delicate question of whether the presence in $\beta X \backslash X$ of a large dyadic set determines $X$ to be pseudocompact is only partly answered: in such case $X$ cannot be Lindelöf. A topological space is said to be dyadic, in a terminology taken from (but not originating with) Engelking and Petczyniski [4], if it is a continuous image of a product of finite discrete spaces. Following Gillman and Henriksen [5], we define $E^{\prime}$-spaces ( $F$-spaces) as those spaces in which disjoint cozero sets (disjoint open $F_{\sigma}$-sets) have disjoint closures.

Among other things Engelking and Petczynski prove that extremally disconnected spaces (= the closure of each open set is open) have no large

Received 19 January 1970. The author appreciates the partial support of the National Science Foundation under Grant NSF-GP-8357. 
dyadic subspaces, a theorem that was subsequently extended to basically disconnected spaces (= the closure of each countable disjoint union of clopen sets is open) by Efimov and Engelking [2]. In Theorem I this result is generalized to $F^{\prime}$-spaces, which need not be totally disconnected and may in fact even be connected. The theorem is downright easy to prove, but its strength may be seen in subsequent applications.

THEOREM 1. Let $X$ be an $F^{\prime}$-space. Then each dyadic subspace of $X$ is finite.

Proof. Since each dyadic space is compact and compact subspaces of $F^{\prime}$-spaces are $F$-spaces, we are reduced to showing that a dyadic $F$-space is finite. Suppose $Y$ is an infinite dyadic $F$-space, and let $f$ be in $C(Y)$ such that the cardinality of $f Y$ is infinite. By a theorem of Corson [1] there exists a compact metric space $Z$ contained in $Y$ such that $f Z=f y$. By $14 \mathrm{~N}$ of [6] $Z$ is finite, so $Y$ is finite.

COROLLARY. If $X$ is any space and $B X$ (= Stone-Čch compactification of $X$ ) is dyadic, then $X$ is pseudocompact.

Proof. Suppose $X$ is not pseudocompact, then there exists a compact $B X-G_{\delta}$ set $U$ contained in $B X \backslash X$. Now $U$ is dyadic by a theorem of Efimov [3], and as $U$ is an $F$-space it must be finite by Theorem 1 . But no finite subset of $\beta X \backslash X$ is a $\beta X-G_{\delta}$ set, so $X$ must be pseudocompact.

This Corollary is actually another substantive result of Engelking and Petczynski. The relatively easy proof is given in order to exploit Theorem 1, which has another application in the proof of Theorem 2. While it is known that any space $Y$ can be represented as $\beta X \backslash X$ for some pseudocompact space $X$ [6], in general such a representation may exist for non-pseudocompact $X$ as well. In other words $B X \backslash X$ need not determine $X$. Conditions on $B X \backslash X$ that determine $X$ are therefore of considerable interest, and dyadicity is such a condition.

THEOREM 2. Let $B X \backslash X$ be dyadic. Then $X$ is pseudocompact.

Proof. Suppose not; then $X$ contains a $C$-embedded copy of the natural numbers $N$. Map $N$ onto the rationals and extend this map to a map $f$ of $B X$ into $\beta R$. Now $f(\beta X \backslash X)$ is a dyadic subset of $\beta R$ and 
meets the compact $G_{\delta}$-set $B R \backslash R$ in an infinite set, which is dyadic. But this violates Theorem $I$, as $\beta R \backslash R$ is an $F$-space. Hence $X$ is pseudocompact.

In particular, Theorem 2 says that if $B X \backslash X$ is a compact metric space then $X$ is pseudocompact. For an example of a pseudocompact locally compact space $X$ such that $B X \backslash X$ is not dyadic, let $X=\beta R \backslash(\beta N \backslash N)$. Then $X$ is pseudocompact. and $B X=\beta R$ [6], but $B X \backslash X=B N \backslash N$ which is an $F$-space, so cannot be dyadic.

COROLLARY. Let $X$ be a locally compact $\sigma$-compact space. Then no infinite compact subset of $B X \backslash X$ is dyadic.

Proof. $B X \backslash X$ is an $F$-space.

One instance of the Corollary is particularly interesting: no infinite compact subset of $\beta N \backslash N$ is dyadic. It would in fact be surprising if any non-pseudocompact space $X$ had an infinite dyadic subset in $B X \backslash X$. This question must be left open, however the result of the Corollary does extend to Lindelöf spaces. One consequence of Theorem 3 to follow is that no metric space having an infinite compact subset, in particular no locally compact non-discrete metric space, can be $B X \backslash X$ for any Lindelöf space $X$. The following easy Lemma is the necessary prelude to Theorem 3 .

LEMMA. If $X$ is Lindelöf, then each compact set contained in $B X \backslash X$ is contained in a compact $B X-G_{\delta}$ set, which is itself contained in $B X \backslash X$.

THEOREM 3. If $X$ is Lindelöf, then no infinite compact subset of $B X \backslash X$ is dyadic.

Proof. Suppose $K$ is a dyadic set in $B X \backslash X$. By virtue of the Lemma there is a compact $\beta X-G_{\delta}$ set $U$ contained in $\beta X \backslash X$ such that $K$ is a subset of $U$. But any compact $B X-G_{\delta}$ set contained in $B X \backslash X$ is an $F$-space, so $K$ must be finite by Theorem 1 .

We now turn to the question of whether an $F^{\prime}$-space can have any dyadic compactifications. Although Engelking and Efimov have characterized the dyadic compactifications of metric spaces as precisely 
those which are metrizable, it is still largely not known which spaces do have dyadic compactifications. We can say that the countable discrete space $N$ has $C$-many dyadic compactifications, for each finite-point compactification is metrizable and hence dyadic, and each uniformly closed subalgebra of $C^{*}(N)$ which has a finite point compactification of $N$ as its maximal ideal space is itself separable metric, and since $C^{*}(N)$ is the uniform closure of an ascending chain of such subalgebras, there must be $C$-many of them as $C^{*}(N)$ is not separable. (We have not necessarily singled out all the dyadic compactifications of $N$ in this way.) It is notable that no other discrete space has any dyadic compactifications at all, and in fact we are able to prove a similar result for any $F^{\prime}$-space, other than $N$ itself.

THEOREM 4. Let $X$ be an $F^{\prime}$-space (not homeomorphic to $N$ ). Then $X$ has no dyadic compactification.

Proof. We may as well assume $X$ to be non-discrete and take $x$ to be a non-isolated point in $X$. Let $Y$ denote a dyadic compactification of $X$ and without loss of generality assume $x_{n}$ to be a sequence in $y \backslash X$ that converges to $x$. Let $f$ be a continuous map from $B X$ to $Y$ that is the identity on $X$ and carries $B X \backslash X$ onto $Y \backslash X$. Let $A$ be the union of the inverse images under $f$ of the points $x_{n}$. Then the closure of $A$ in $X$ is $A \cup\{x\}$ and $A$ is $\sigma$-compact, hence $C^{*}$-embedded in its closure as $B X$ is an $F$-space. We have that $B A=A \cup\{x\}$, so $A$ is necessarily pseudocompact. But this is not possible, so $X$ can have no dyadic compactification.

\section{References}

[1] H.H. Corson, "Normality in subsets of product spaces", Amer. J. Math. 81 (1959), 785-796.

[2] B. Efimov and R. Engelking, "Remarks on dyadic spaces II", Colloq. Math. 13 (1965), 181-197.

[3] B. Efimov; "Dyadic bicompacta", Soviet Math. Dok2. 4 (1963), 496-500. 
[4] R. Engelking and A. Pelczynski, "Remarks on dyadic spaces", Cozzoq. Math. 11 (1963), 55-63.

[5] Leonard Gillman and Melvin Henriksen, "Rings of continuous functions in which every finitely generated ideal is principal", Trans. Amer. Math. Soc, 82 (1956), 366-391.

[6] Leonard Gillman and Meyer Jerison, Rings of continuous functions (Van Nostrand, Princeton, New Jersey, 1960).

Wesleyan University,

Middletown,

Connecticut, USA. 